Revista Española de la Transparencia

Núm. 7. Segundo Semestre 2018

ISSN 2444-2607. Págs. 99-123
Alberto Prado Román 1

Paola Plaza Casado²

Sandra Escamilla Solano 3

Ana Cruz Suárez ${ }^{4}$

Universidad Rey Juan Carlos

\title{
Identificación de la transparencia como una herramienta de diferenciación en el sector bancario español
}

\footnotetext{
1 Profesor Titular de Universidad Interino del Departamento Economía de la Empresa de la Universidad Rey Juan Carlos. Doctor en Marketing por la Universidad Rey Juan Carlos. Miembro de AEDEM y MBERG.

2 Profesor Ayudante Doctor del Departamento Economía de la Empresa de la Universidad Rey Juan Carlos. Doctora en Economía Financiera y Contabilidad por la Universidad Rey Juan Carlos. Miembro de AEDEM y MBERG.

3 Profesor Ayudante Doctor del Departamento Economía de la Empresa de la Universidad Rey Juan Carlos. Doctora en Economía Financiera y Contabilidad por la Universidad Rey Juan Carlos. Miembro de AEDEM y MBERG.

4 Profesor Ayudante Doctor del Departamento Economia de la Empresa de la Universidad Rey Juan Carlos. Doctora en Dirección de Empresas por la Universidad Rey Juan Carlos. Miembro de AEDEM y MBERG.
} 


\section{Alberto Prado Román, Paola Plaza Casado, Sandra Escamilla Solano y Ana Cruz Suárez}

RESUMEN: Durante años, la crisis económica que asoló mercados enteros ha provocado que los consumidores se consideren engañados por todas las empresas. Éstos consideran que las empresas, no solo durante el período de crisis, pero acentuado por éste, no tenían ningún reparo en llevar acciones discutibles si con ello alcanzaban sus propios objetivos. Consideran además, que lejos de que la información que transmiten las empresas sea transparente, éstas ocultan más información que de conocerse, los consumidores podrian cambiar no solo su opinión respecto a la imagen de las mismas, sino sus decisiones comerciales. Dentro de este escenario, uno de los sectores que ha sido considerado en los últimos años como menos transparente en España es el sector financiero, destacando dentro de éste el sector bancario. Sin embargo, existe en España una fuerte restricción legislativa que obliga a dichas entidades a ser transparentes. Ante esto, nuestra investigación profundiza en el concepto de transparencia, seleccionando una serie de indicadores que nos permitan determinar el nivel de transparencia de dicho sector, y demostrar si el escepticismo de los consumidores hacia éstas está bien justificado. Para ello, hemos aplicado dichos indicadores analizando las memorias de las principales entidades bancarias de España, determinando que aunque su nivel de transparencia es muy alto, los consumidores no lo perciben así. Provocando que dichas entidades tengan que plantear nuevas estrategias de imagen para poder llegar a los consumidores, y convencerles de su nivel de transparencia es elevado y con ello asegurarse la legitimidad de los mismos.

PALABRAS CLAVE: Transparencia, sector bancario, confianza, responsabilidad social, sector financiero.

CONTENIDOS: 1. Introducción. - 2. Marco conceptual. - 2.1. El papel de la transparencia en la empresa. - 2.2. La responsabilidad social empresarial como elemento potenciador de la transparencia - 2.3. Medición de la transparencia. - 3. Metodología. - 4. Resultados. - 5. Discusión, conclusiones e implicaciones. Bibliografía. 


\section{Identificación de la transparencia como una herramienta de differenciación en el sector bancario español}

\section{Identification of transparency as a differentiation tool in the spanish banking sector}

ABSTRACT: For years, the economic crisis that devastated entire markets has caused consumers to consider themselves cheated by all companies. They consider that the companies, not only during the crisis period, but accentuated by it, did not have any objection to take debatable actions if they achieved their own objectives. They also consider that far from the fact that the information transmitted by companies is transparent, they hide more information than knowing each other, consumers could change not only their opinion regarding their image, but also their commercial decisions. Within this scenario, one of the sectors that has been considered in recent years as less transparent in Spain is the financial sector, highlighting within this the banking sector. However, there is a strong legislative restriction in Spain that forces to these entities to be transparent. Given this, our research deepens the concept of transparency, selecting a series of indicators that allow us to determine the level of transparency of this sector, and demonstrate the consumers' skepticism is well justified. To do this, we have applied these indicators analyzing the reports of the main banks in Spain, determining that although their level of transparency is very high, consumers do not perceive it that way. This has cause that these entities have to pose new image strategies to reach consumers and convince them of their level of transparency is high and thereby ensure the legitimacy of them.

KEYWORDS: Transparency, bank sector, confidence, social responsibility, financial sector. 


\section{Alberto Prado Román, Paola Plaza Casado, Sandra Escamilla Solano y Ana Cruz Suárez}

\section{INTRODUCCIÓN.}

La organización Transparencia Internacional España asume el compromiso de incrementar el nivel de información y apertura de las distintas instituciones públicas para mejorar el conocimiento de la población. Para ello, elabora distintos informes sobre la trasparencia de distintos ámbitos, como la corrupción, el sector público, sector privado, fútbol..., desarrollando nueve índices para su análisis5. Sin embargo de los nueve índices que elabora, éstos se centran en el análisis de la transparencia en ayuntamientos, Comunidades Autónomas, gestión de agua y diputaciones y Parlamentos, clubs de fútbol, federaciones deportivas, empresas públicas y partidos políticos. La relevancia del objeto de estudio de estos índices es claramente relevante y necesaria. Pero también se detecta la necesidad, y su importancia, en analizar el nivel de transparencia de las instituciones financieras. Detectándose la ausencia, tanto de estudios e informes al respecto.

A pesar de que la organización Transparencia Internacional España no contempla un índice en el que se analizan las instituciones financieras, el barómetro de la transparencia analiza el sector bancario. Sin embargo, no profundiza en la transparencia de las instituciones bancarias. Según el Barómetro de la transparencia de España, encuesta de opinión pública, en los informes realizados en los años 2015 y 2016 por Acreditra6 (Asociación Española de Profesionales de la Transparencia), se pueden extraer una serie de conclusiones sobre la percepción que tienen los encuestados sobre la transparencia de distintas instituciones. Se constata que para más del $90 \%$ de los encuestados, la transparencia en la vida pública y social es importante o muy importante. Además, en 2015, un 71,5\% considera que la trasparencia puede ayudar a prevenir la corrupción, incrementándose ese porcentaje el año siguiente hasta llegar a un 76,2\%. La existencia de una Ley de transparencia es ampliamente valorada (79,8\% en el año 2015 y 50,5\% en el año 2016) por los encuestados. No obstante, a pesar del respaldo mostrado a esta Ley, los porcentajes disminuyen cuando se pregunta por la obligación de la transparencia en la gestión pública (65,2\% afirma conocer que esta obligación existe). A la hora de trasladar de manera más específica la valoración de la transparencia de las instituciones públicas en el año 2015, más del $70 \%$ de los encuestados la valora por debajo del 5 (siendo 10 el valor de mayor transparencia y o el menor). Uno de los problemas que se vislumbra, a raíz de las preguntas formuladas en el Barómetro, es que más del 70\% de los encuestados apenas confían en la información publicada por las Administraciones Públicas

\footnotetext{
${ }^{5}$ Estos indices están disponibles en https://transparencia.org.es/indices-transparencia-espana/

${ }^{6}$ Principales resultados recogidos en el Segundo Barómetro de la Transparencia de España 2016 desarrollado por Acreditra. Resultados disponibles en: http://acreditra.com/barometro-de-latransparencia-de-espana-2016/
} 


\section{Identificación de la transparencia como una herramienta de differenciación en el sector bancario español}

respecto a sus obligaciones de transparencia. Además, en el Barómetro se pide a los encuestados que valoren, especificamente, el nivel de transparencia de partidos políticos ( $97 \%$ piensa que prácticamente ninguna), sector bancario $(94,4 \%$ piensa que prácticamente ninguna), sindicatos (90\% ninguna), organizaciones empresariales (86,7\% ninguna) y medios de comunicación (65,9\% ninguna).

En los últimos años, la transparencia ha incrementado su importancia tanto en las instituciones públicas, gobiernos, corporaciones como en las empresas. La transparencia es algo positivo para la empresa y también para la sociedad que se relaciona con la misma. Un gran beneficio que obtendrá la empresa de ser transparente es la posesión de información oportuna y efectiva que facilitará su desarrollo económico y comercial (Ochsenius, 2017). Otro de los beneficios de ser una empresa transparente es la proyección de una imagen de confianza y de veracidad de cara al exterior, tanto a nivel responsable y sostenible, como respecto a las restantes actividades económicas y comerciales. Por último, debemos destacar la relevancia de la transparencia debido a que es considerada como antecedente a la generación, tanto de credibilidad y legitimidad, como de reputación y creación de valor para la empresa (Baraibar Díez y Luna Sotorrío, 2018).

De este modo, se detecta la necesidad de profundizar en el análisis del nivel de transparencia de las entidades bancarias que operan en el mercado español. Por ello, esta investigación busca alcanzar los siguientes objetivos: 1) Analizar si las entidades bancarias que operan en el mercado español son realmente transparentes. 2) Analizar las implicaciones del nivel de transparencia de las entidades bancarias sobre el mercado y determinar las estrategias que deberán implantar estas entidades para mostrar una imagen transparente al mercado.

\section{MARCO CONCEPTUAL.}

\subsection{El papel de la transparencia en la empresa.}

El concepto de transparencia se caracteriza por una elevada complejidad, por ello no podemos entender que el nivel de transparencia de la empresa esté sujeto únicamente a la rendición de sus cuentas. Así, Marcuello et al. (2007) identifican a la transparencia como la capacidad de la empresa para estar disponible y susceptible de aportar información. De este modo, la empresa no solo deberá rendir sus cuentas, sino que deberá caracterizarse por una actitud voluntariosa y clara en el proceso de rendición de sus cuentas. En esta línea se muestra Villanueva (2011), que entiende la transparencia como la posición asumida por las entidades, ya sean públicas o privadas, de comportarse en base a los estándares éticos y cuyos procesos y resultados puedan ser presentados a todas aquellas personas que precisen de su conocimiento. Esto implica que la empresa no podrá hacer distinciones entre las partes involucradas o afectadas en su toma de decisiones en función de la importancia que tengan para la misma. Si la empresa estimase que el 


\section{Alberto Prado Román, Paola Plaza Casado, Sandra Escamilla Solano y Ana Cruz Suárez}

nivel de transparencia depende del impacto que tuviesen los diferentes stakeholders sobre su actividad, se podría mostrar especialmente transparente con sus inversores, pero muy poco con la sociedad. Por ello, Ruiz et al. (2008) defienden que la necesidad de ser transparentes no puede ser únicamente identificada en los inversores, ya que las decisiones de la empresa influirán, en mayor o menor medida, en todos los stakeholders de la misma.

En base a lo anteriormente expuesto, y basándonos en Gálvez et al. (2009), podemos definir la transparencia como el conjunto de mecanismos que tiene como funciones principales la verificación y evaluación de actuaciones empresariales y el análisis y comunicación de la información segura y veraz por parte de los distintos stakeholders de la empresa.

Respecto a la relevancia de la transparencia en el ámbito empresarial, y a pesar de que actualmente el estudio del nivel de transparencia de las distintas entidades que operan en el mercado ha aumentado en atención (Deegan y Rankin, 1996; Gray et al., 1995; Hooghiemstra, 2000; Kolk, 2003; Gálvez et al., 2009; Arredondo et al., 2014; García et al., 2016), podemos observar que muchas de las investigaciones sobre transparencia se centran en el sector público o con fines no lucrativos.

La transparencia es un elemento de enorme relevancia no solo en el ámbito público, sino que también lo es en el ámbito empresarial, ya que se ha identificado como generador tanto de reputación (Baraibar Díez y Luna Sotorrio, 2012) como de confianza (Arredondo et al., 2014). Especialmente en los mercados financieros, ya que es necesario que la empresa transmita toda la información relevante, correcta y veraz a los posibles inversores y que su transmisión sea simétrica, equitativa y en tiempo real (Barea, 2004).

Para que la transparencia pueda ser considerada como generadora de confianza, la empresa deberá ser capaz de dar respuesta a todas las cuestiones planteadas por cualquiera de sus stakeholders sobre el funcionamiento de la misma. Debemos tener en cuenta que la mayor accesibilidad a la información de la empresa permitirá aumentar las posibilidades de que los accionistas e inversionistas, entre otros, puedan tomar decisiones más convenientes (Kliksberg, 2003). Esto se debe a que la unión entre los conceptos de transparencia y de información han sido capaces de reducir la asimetría existente entre la dirección de la empresa y la información relativa a la toma de decisiones que conocían los distintos stakeholders (García et al., 2016).

Por ello, el objetivo de la dirección de la empresa deberá ser generar un elevado nivel de transparencia motivado por la necesidad y exigencia de tener disponible la información de la misma para los distintos stakeholders (Rivero, 2005). Así, podrá aumentar la confianza del inversor, y del resto de stakeholders, mostrando que las decisiones de la empresa no solo buscan alcanzar objetivos meramente económicos o a corto plazo, sino que se toman decisiones sostenibles y que puedan influir positivamente en los distintos stakeholders de la empresa. Además, 


\section{Identificación de la transparencia como una herramienta de differenciación en el sector bancario español}

un mayor nivel de transparencia permitirá que la empresa pueda gozar de una mayor valoración, reducir los costes de inversión y de una mayor capacidad de los inversores para centrar sus decisiones de inversión en esta empresa, así como, mejorar su reputación en el mercado (Gibbins et al., 1990; Lev, 1992; Skinner, 1994; Watson et al., 2002, Baraibar Díez y Luna Sotorrío, 2012).

\subsection{La responsabilidad social empresarial como elemento potenciador de la transparencia.}

La globalización ha suscitado un crecimiento espectacular de las interrelaciones de los agentes que operan en el mundo, teniendo como principal consecuencia el aumento de poder de las grandes multinacionales. El papel de las nuevas tecnologías (Prado, 1995), la inclusión de los valores éticos en la estrategia de la empresa (Bañon et al., 2011), la necesidad de aumentar la reputación y valorar al capital humano (Lipovetsky, 2006), así como, la necesidad de divulgar todas las acciones y medidas que llevan a cabo (Escamilla et al., 2016), implican que el paradigma empresarial cambie.

A la hora de abordar la Responsabilidad Social Corporativa (RSC), se detecta la existencia de numerosas definiciones y teorías, lo cual nos lleva a encontrarnos ante una gran falta de consenso (Garriga y Melé, 2004; Alvarado et al., 2011). La RSC se centra "en la integración voluntaria por parte de la empresa, tanto en su forma de gobierno como de gestión, de las preocupaciones sociales, medioambientales, económicas y del respeto de los Derechos Humanos derivadas de las relaciones que mantiene la empresa con sus grupos de interés directos, así como con cualquier persona que se vea afectada por el desarrollo de la actividad económica de la misma" (Escamilla et al, 2013). En definitiva, se trata de asumir una responsabilidad que va más allá de lo legalmente exigido y que esté enfocada en mejorar el bienestar social y económico de la empresa, así como, del entorno que las rodea. Quienes realmente hacen posible una evolución de la propia concepción de la RSC son los stakeholders que se ven afectados por las empresas (Freeman, 1984). Por un lado, los accionistas, quienes exigen cada vez una mayor transparencia en las actuaciones que realiza la empresa con el objetivo de trasladar confianza a la empresa y al propio mercado; por otro lado, los clientes, quienes solicitan día a dia productos y servicios de calidad, innovadores y con precios justos (Kliksberg, 2006); y por último, la sociedad, consciente que para que la empresa tenga éxito la RSC no puede mantenerse al margen.

El rol de las empresas - sociedad debe ir en consonancia con el modelo económico elegido, considerando a la empresa como un ente social (Navarro, 2008) que está en contacto con su entorno. En este contexto, la aplicación de politicas de RSC se justifica por el hecho de que ayuda a resolver conflictos y a distribuir el valor creado entre los distintos grupos. Así, las empresas socialmente responsables obtendrán efectos positivos futuros que permitirán asegurar la sostenibilidad, tanto de la empresa, como de la sociedad, (Porter y Kramer, 2003), 


\section{Alberto Prado Román, Paola Plaza Casado, Sandra Escamilla Solano y Ana Cruz Suárez}

reforzar su posición competitiva, motivado por la mayor aceptación de su imagen y, por tanto, minimizar su riesgo reputacional (Porter y Kramer, 2003; Méndez, 2005; Valderrama, 2007), adquirir ventajas competitivas (Cortés, 2008; Olmedo y Martínez, 2011) y ayudar a aumentar su legitimidad (Diez et al., 2014). En esta línea, destaca el informe sobre "La gestión de los riesgos de corrupción en las empresas del IBEX 35", desarrollado por el Observatorio de Responsabilidad Social Corporativa (2019), en el que se identifica que únicamente una entidad del sector financiero perteneciente al IBEX 35 (Caixabank) cuenta con una política pública específica en materia de corrupción. Aunque el cumplimiento de esta política no se extiende a sus proveedores y socios comerciales.

Las empresas deben saber interactuar en un mundo global donde las exigencias de la ciudadanía pasarán por evaluar y juzgar sus actuaciones sociales, medioambientales y económicas, al ser mucho más exigentes como consecuencia de los escándalos vividos, tanto financieros, como empresariales, en las últimas décadas (Baumann, 2016). Una forma de reducir esa posible brecha de confianza es la utilización de la transparencia corporativa, como vehículo conductor para comunicar no solo el desempeño financiero, sino también toda la información relativa a RSC. Siempre se ha considerado que la transparencia es una variable a tener en cuenta cuando se analiza la divulgación de la información de RSC. valorándose positivamente ese compromiso por parte de la empresa de comunicar las acciones de la empresa a los grupos de interés (Alejos, 2015). Son indudables los beneficios que genera la divulgación de información para la toma de decisiones de la empresa (Piechocki, 2004; Toms, 2002), pero al igual que no existe consenso sobre que es o no RSC, cuando analizamos el nivel de divulgación de la información de la empresa se tiende a confundir con ser más o menos transparentes. Baraibar-Diez y Luna (2018), en su investigación, revelan que existe un problema al confundir la transparencia con la divulgación, entendiendo la transparencia como algo que va más a allá de divulgar. Por ello, se debe entender la doble contribución que tiene la transparencia dentro de las empresas, por un lado, debe comunicar la realidad de la empresa para establecer lazos de confianza mutua, es decir, entenderla no como un "fin sino como un medio para conseguir un entorno de confianza" (Cabrera, 2008, 7) ${ }^{7}$ y, por otro lado, que "a través de la transparencia la empresa incremente su desempeño social" (Alejos, 2015, 7). Por último, debemos señalar que, dentro de las prácticas llevadas a cabo por las empresas responsables y sostenibles, la práctica de buen gobierno en la empresa es identificada como esencial en el establecimiento de un adecuado nivel de transparencia (Canales y Romero, 2017). Entendiendo como buen gobierno "un conjunto de recomendaciones acerca de la apropiada estructura de los órganos de gobierno y del adecuado comportamiento de los miembros de esos órganos" (Aguilera y Cuervo, 2004).

\footnotetext{
${ }^{7}$ Citado en Alejos (2015)
} 


\section{Identificación de la transparencia como una herramienta de diferenciación en el sector bancario español}

\subsection{Medición de la transparencia.}

Con el fin de analizar el nivel de transparencia tanto de las administraciones públicas como de instituciones privadas, en diciembre de 2013 nació la Asociación Española de Profesionales de la Transparencia (Acreditra). Esta organización sin ánimo de lucro agrupa tanto a los profesionales del sector de la consultoría y auditoría como a todas aquellas entidades que asumen y se comprometen con los estándares más altos de calidad. Entre los objetivos propuestos por Acreditra, podemos destacar, entre otros, la promoción de la Transparencia, el Buen Gobierno y el Gobierno Abierto y la evaluación y acreditación de la Transparencia, tanto de administraciones públicas, como de instituciones privadas.

Para poder analizar y evaluar el grado de transparencia de las administraciones públicas, como de instituciones privadas, se diseñó el Sistema Español de Acreditación de la Transparencia (SIESTRA). Este sistema de acreditación se basa en siete pilares que suponen la base del mismo: Credibilidad, Exigencia, Utilidad, Prestigio, Rigurosidad, Autoexigencia y Amplitud de Alcance. Además, el SIESTRA utiliza 149 indicadores que le permitirán analizar el grado de transparencia de las administraciones públicas e instituciones privadas que operan en el mercado español. Los 149 indicadores se distribuyen en función del ámbito de análisis y se agrupan de la siguiente manera:

- Publicidad Activa. Está compuesto por 119 indicadores y se analiza el grado de transparencia en función de la información general de la entidad, de los órganos de gobierno y cargos de representación/dirección, organización y recursos humanos, relaciones con la ciudadanía y los grupos de interés, contratación, información económico-financiera y patrimonio general, detalles sobre ingresos y gastos relevantes, planificación, gestión y evaluación, normativa, urbanismo, obras y medio ambiente y controles formales.

- Derecho de acceso a la información pública. Está compuesto por 8 indicadores y se analiza el grado de transparencia en función de la organización de la satisfacción del Derecho y la satisfacción del Derecho.

- Organización transparente. Está compuesto por 22 indicadores y se analiza el grado de transparencia en función del compromiso y principios generales de transparencia y buen gobierno, organización para la transparencia y el buen gobierno y obligaciones derivadas de la acreditación.

A pesar del diseño de una herramienta completa y eficaz para medir el grado de transparencia de la entidad objeto de estudio, se detecta que la utilización de una misma herramienta para acreditar tanto a administraciones públicas como a instituciones privadas, no es del todo aplicable. Esto se debe a que hay determinados factores que no permiten un adecuado análisis de la transparencia 


\section{Alberto Prado Román, Paola Plaza Casado, Sandra Escamilla Solano y Ana Cruz Suárez}

de las instituciones privadas respecto a las administraciones públicas y entre las instituciones privadas. Además, en el estudio de la transparencia de las instituciones privadas se incluyen a los distintos sectores que componen el mercado, dificultando la identificación del grado de transparencia de las instituciones privadas en función de los sectores. Por ello, se ha decidido analizar y medir el grado de transparencia del sector bancario a través de 50 indicadores del sistema SIESTRA. La elección de estos indicadores se llevó a cabo analizando las características específicas que definen al sector bancario español y a la identificación de aquellos indicadores que mejor se adaptasen al mismo. A pesar de que en el sistema SIESTRA se utilizan indicadores adecuados para analizar a distintos tipos de entidades, como es el caso de las entidades gubernamentales, es la propia muestra del estudio la que obligó a la identificación y valoración de indicadores que mejor se adaptasen a la investigación. Por ello, se procedió a escoger aquellos indicadores que, por su naturaleza, permitiesen realizar un análisis coherente y profundo de la capacidad de transparencia del sector bancario español.

Tras analizar la adecuación de los indicadores para nuestra investigación, se procedió a agrupar los indicadores escogidos, en función del ámbito de análisis, en siete categorías diferentes. La primera categoría (Tabla 1), recoge indicadores de análisis de la información de los bancos y de sus órganos de gobierno. Este grupo de indicadores tienen como función principal determinar el grado de conocimiento que se aporta al mercado respecto a los elementos constitutivos de los bancos objetos de análisis. La segunda categoría (Tabla 2), se centra en el análisis de la organización y recursos humanos de los bancos objetos de estudio. A través de estos indicadores se analiza si los bancos de la muestra, realmente, aportan información relevante sobre la organización y funciones de los distintos departamentos que la componen. La tercera categoría de indicadores (Tabla 3) tiene como función determinar si los bancos muestran un comportamiento transparente en sus relaciones, tanto con sus clientes, como los restantes grupos de interés. Los indicadores de la cuarta categoría (Tabla 4) se encargan de evaluar si la información patrimonial y económica de la empresa es fiel a la realidad. Estos indicadores no analizan la veracidad de la información contable, ya que esta labor es competencia de los auditores, sino que determinarán si los bancos aportan la información de sus estados al mercado. La quinta categoría (Tabla 5) está compuesta por indicadores cuya función es analizar si las labores de planificación, gestión y evaluación de los recursos de los bancos se ofrecen de forma coherente, sin irregularidades. La sexta categoría (Tabla 6) contiene indicadores que buscan analizar la existencia de normativa que rija a los bancos y el registro de acciones de auditoría de sus actividades. Por último, la categoría siete (Tabla 7), contiene indicadores que determinarán el grado de compromiso de los bancos en la aplicación de los principios generales de transparencia y buen gobierno. 


\section{Identificación de la transparencia como una herramienta de differenciación en el sector bancario español}

TABLA 1. Indicadores de análisis de la información de los bancos y de sus órganos de gobierno

\begin{tabular}{|l|l|}
\hline \multicolumn{2}{|c|}{ INFORMACIÓN DE LA ENTIDAD Y ÓRGANOS DE GOBIERNO } \\
\hline 1 & Denominación y datos básicos de la entidad \\
\hline 2 & Objeto y funciones de la entidad \\
\hline 3 & $\begin{array}{l}\text { Relación de las entidades públicas y privadas en las que la entidad tiene } \\
\text { participación societaria }\end{array}$ \\
\hline 4 & Relación de otras entidades a las que se pertenece \\
\hline 5 & Enumeración de los órganos de gobierno \\
\hline 6 & $\begin{array}{l}\text { Relación nominal de las personas integrantes de los órganos de gobierno } \\
\text { y dirección }\end{array}$ \\
\hline 7 & $\begin{array}{l}\text { Currículum actualizado de las personas integrantes de los órganos de } \\
\text { gobierno y dirección }\end{array}$ \\
\hline 8 & Reuniones y acuerdos tomados por los órganos de Gobierno \\
\hline
\end{tabular}

TABLA 2. Indicadores de análisis de la organización y recursos humanos de los bancos

\begin{tabular}{|c|l|}
\hline \multicolumn{2}{|c|}{ ORGANIZACIÓN Y RECURSOS HUMANOS } \\
\hline 9 & Organigrama general de la entidad \\
\hline 10 & Funciones de los departamentos \\
\hline 11 & Contacto de los departamentos \\
\hline \multicolumn{2}{|c|}{ Fuente: Elaboración propia basado en SIESTRA (2016) } \\
\hline
\end{tabular}

TABLA 3. Indicadores de análisis de las relaciones de los bancos con los clientes y grupos de interés

\begin{tabular}{|c|l|}
\hline \multicolumn{2}{|c|}{ RELACIONES CON LOS CLIENTES Y GRUPOS DE INTERÉS } \\
\hline 12 & Horarios de atención \\
\hline 13 & Webs y Portales electrónicos \\
\hline 14 & Precios e importe de los servicios y tasas \\
\hline 15 & Información sobre los órganos de participación estructurada \\
\hline 16 & Información sobre los procesos participativos \\
\hline 17 & Guía de Procedimientos y Trámites y/o Catálogo de Servicios \\
\hline 18 & Cartas de Servicios \\
\hline 19 & Quejas y sugerencias \\
\hline \multicolumn{2}{|c|}{ Fuente: Elaboración propia basado en SIESTRA (2016) } \\
\hline
\end{tabular}

Fuente: Elaboración propia basado en SIESTRA (2016) 


\section{Alberto Prado Román, Paola Plaza Casado, Sandra Escamilla Solano y Ana Cruz Suárez}

TABLA 4. Indicadores de análisis de la información económico-financiera y patrimonio general de los bancos

\begin{tabular}{|l|l|}
\hline \multicolumn{2}{|c|}{ INFORMACIÓN ECONÓMICO-FINANCIERA } \\
Y PATRIMONIAL GENERAL \\
\hline 20 & Presupuestos completos \\
\hline 21 & Cuentas anuales \\
\hline 22 & Endeudamiento \\
\hline 23 & Deuda financiera \\
\hline 24 & Evolución del endeudamiento \\
\hline 25 & Ratios de endeudamiento \\
\hline 26 & Plazo de pago a proveedores \\
\hline 27 & Ratios económico-financieros \\
\hline 28 & Inventario de Bienes y Derechos \\
\hline 29 & Inmuebles \\
\hline 30 & Bienes especiales \\
\hline 31 & Informe de sostenibilidad financiera \\
\hline
\end{tabular}

Fuente: Elaboración propia basado en SIESTRA (2016)

TABLA 5. Indicadores de análisis del nivel de planificación, gestión y evaluación de los bancos

\begin{tabular}{|l|l|}
\hline \multicolumn{2}{|c|}{ PLANIFICACIÓN, GESTIÓN Y EVALUACIÓN } \\
\hline 32 & $\begin{array}{l}\text { Datos estadísticos e indicadores socioeconómicos sobre su ámbito } \\
\text { territorial o sectorial de actuación }\end{array}$ \\
\hline 33 & Informes de diagnóstico \\
\hline 34 & Plan o Programa general de la entidad \\
\hline 35 & Memoria general de la entidad \\
\hline 36 & Informe sobre el coste y rendimiento de los servicios \\
\hline 37 & Informes sobre la calidad y evaluación de servicios \\
\hline 38 & Indicadores de eficacia y eficiencia \\
\hline
\end{tabular}

Fuente: Elaboración propia basado en SIESTRA (2016)

TABLA 6. Indicadores de análisis de la normativa y controles formales de los bancos

\begin{tabular}{|l|l|}
\hline \multicolumn{2}{|c|}{ NORMATIVA Y CONTROLES FORMALES } \\
\hline 39 & Normativa básica de organización y funcionamiento \\
\hline 40 & Normativa generada por la entidad \\
\hline 41 & Informes internos de Intervención y Auditoría de Cuentas \\
\hline 42 & Informes externos de auditoria y/o fiscalización \\
\hline \multicolumn{2}{|c|}{ Fuente: Elaboración propia basado en SIESTRA (2016) }
\end{tabular}




\section{Identificación de la transparencia como una herramienta de differenciación en el sector bancario español}

\section{TABLA 7. Indicadores de análisis del compromiso y principios generales de transparencia y buen gobierno de los bancos}

\begin{tabular}{|l|l|}
\hline \multicolumn{2}{|c|}{\begin{tabular}{c|} 
COMPROMISO Y PRINCIPIOS GENERALES \\
DE TRANSPARENCIA Y BUEN GOBIERNO
\end{tabular}} \\
\hline 43 & Código de Buen Gobierno o Código Ético \\
\hline 44 & Régimen sancionador en materia de Buen Gobierno \\
\hline 45 & Normativa reguladora de transparencia \\
\hline 46 & Web amigable y comprensible \\
\hline 47 & Gratuidad del acceso a la información sobre transparencia \\
\hline 48 & Publicación en la web de noticias de interés \\
\hline 49 & Igualdad de género \\
\hline 50 & Proyectos de actuación de responsabilidad social \\
\hline \multicolumn{2}{|c|}{ Fuente: Elaboración propia basado en SIESTRA (2016) } \\
\hline
\end{tabular}

\section{METODOLOGÍA}

El escenario de estudio de la investigación es el sector bancario español. El motivo que nos ha impulsado a analizar el grado de transparencia de este sector no es solo su gran relevancia en la economía española, sino también se debe a los distintos problemas que ha tenido este sector y que ha provocado que la sociedad se muestre recelosa. Maudos (2012) argumenta que los problemas del sector bancario español se deben a la época de expansión económica, destacando como principales causantes a prácticas como abundancia de liquidez, al acelerado crecimiento del endeudamiento o al elevado ritmo de crecimiento del crédito bancario, entre otros. Además, debemos tener en cuenta que España es uno de los pilares en el funcionamiento y en el proceso económico de la Unión Europea y por ello relevante como objeto de nuestra investigación.

Así, se identifica que las posibles problemáticas financieras que afecten a España también afectarán a todos los países miembros de la Unión Europea y con ello al resto de paises del mundo que establecen acuerdos comerciales con la Unión Europea. Como ocurrió cuando en junio de 2012 España solicitó formalmente financiación a la Unión Europea para superar la crisis que afectó al sector financiero español (GESEM, 2012)8.

Para determinar el grado de transparencia de este sector se ha procedido a identificar aquellos bancos que se encuentran dentro del IBEX - 35 y cuyo nivel de capitalización tenía un nivel significativo. Se han tenido en cuenta solo los bancos que se encuentran en el IBEX - 35 y no todos los bancos que operan en el

\footnotetext{
${ }^{8}$ Análisis con título "Rescate para el Sistema Financiero Español" realizado por GESEM Asesoramiento Financiero EAFI, S.L. Disponible en: http://www.gesemav.com/informes/historico/5Rescate\%2opara\%20el\%20sistema\%20financiero\%20espa\%C3\%B10l.pdf
} 


\section{Alberto Prado Román, Paola Plaza Casado, Sandra Escamilla Solano y Ana Cruz Suárez}

mercado español porque las entidades bancarias que pertenecen a este índice cuentan con mayor liquidez, cotizando en el Sistema de Interconexión Bursátil Electrónico (SIBE). Además, dentro de este grupo de bancos solo se han seleccionado aquellas entidades que estuviesen entre las primeras 10 empresas del IBEX - 35 y cuyo capitalización, en junio de 2018, fuese notablemente mayor que las restantes entidades bancarias. Así, se han escogido como objeto de muestra al Banco Santander (74.105.285), al BBVA (39.707.265) y al Caixabank (21.425.511)9. De este modo, entendemos que estos tres bancos son los referentes del mercado bancario español y que nos permitirán evaluar el grado de transparencia del mismo y sus repercusiones tanto en el mercado como en la sociedad. Para analizar el grado de transparencia del sector bancario español se analizará la información disponible, en el año 2018, en las memorias de los bancos objetos de estudio.

\section{RESULTADOS}

Demostrada la relevancia de la investigación y la metodología aplicada en la investigación, procedemos a explicar los resultados obtenidos ${ }^{10}$. Como se ha visto anteriormente, los indicadores de transparencia bancaria, un total de 50, miden diferentes aspectos de las entidades y por ello vamos a proceder a analizarlos en función de cada uno de las categorías.

Para explicar de manera clara todos los resultados se ha procedido a presentar todos los indicadores agrupados en diferentes categorías mediante tablas, en función de la parte de la empresa que se pretende medir, y justo debajo la explicación de los resultados principales que se derivan de las mismas. Por ello, en primer lugar se procederá a analizar los indicadores que hacen referencia a los datos de las entidades y los respectivos órganos de gobierno:

\footnotetext{
${ }^{9}$ El valor de capitalización de cada banco ha sido obtenido del mercado de valores el 26 de junio de 2018 y está expresado en millones de euros.

${ }^{10}$ Los resultados expuestos en esta investigación han sido obtenidos a través del análisis de las memorias de los bancos objetos de estudio: Banco Santander (disponible en: https://wWw.santander.com/csgs/Satellite/CFWCSancomQP01/es_ES/Corporativo/Accionistas-eInversores.html), BBVA (disponible en: https://accionistaseinversores.bbva.com/informacionfinanciera/informes-financieros/\#2018), Caixabank en: https://www.caixabank.com/informacionparaaccionistaseinversores/gobiernocorporativo/juntagene ralaccionistas/2013_es.html).
} 


\section{Identificación de la transparencia como una herramienta de differenciación en el sector bancario español}

TABLA 8. Información de la entidad y órganos de gobierno

\begin{tabular}{|c|c|c|c|c|}
\hline \multicolumn{2}{|r|}{$\begin{array}{c}\text { INFORMACIÓN DE LA ENTIDAD } \\
\text { Y ÓRGANOS DE GOBIERNO }\end{array}$} & SANTANDER & BBVA & CAIXABANK \\
\hline 1 & Denominación y datos básicos de la entidad & $x$ & $X$ & $X$ \\
\hline 2 & Objeto y funciones de la entidad & $\mathrm{x}$ & $\mathrm{x}$ & $x$ \\
\hline 3 & $\begin{array}{l}\text { Relación de las entidades públicas y privadas en } \\
\text { las que la entidad tiene participación societaria }\end{array}$ & $x$ & $x$ & $x$ \\
\hline 4 & $\begin{array}{l}\text { Relación de otras entidades a las que se } \\
\text { pertenece }\end{array}$ & $x$ & $x$ & $x$ \\
\hline 5 & Enumeración de los órganos de gobierno & $\mathrm{x}$ & $\mathrm{x}$ & $x$ \\
\hline 6 & $\begin{array}{l}\text { Relación nominal de las personas integrantes de } \\
\text { los órganos de gobierno y dirección }\end{array}$ & $x$ & $x$ & $x$ \\
\hline 7 & $\begin{array}{l}\text { Curriculum actualizado de las personas } \\
\text { integrantes de los órganos de gobierno y } \\
\text { dirección }\end{array}$ & $x$ & $x$ & $x$ \\
\hline 8 & $\begin{array}{l}\text { Reuniones y acuerdos tomados por los órganos } \\
\text { de Gobierno }\end{array}$ & $x$ & $x$ & $x$ \\
\hline
\end{tabular}

Fuente: Elaboración propia.

Como se puede observar en la tabla 8, las principales entidades bancarias del mercado español cumplen con los 8 indicadores de transparencia de la organización. Dentro de esta categoría destaca que las tres muestran de manera fácil y accesible la información de todos los miembros de sus respectivos órganos de gobierno, y también se muestra de manera muy clara las remuneraciones de cada uno de los miembros de éstos mediante tablas donde se detallan las cantidades que perciben y los conceptos de dichas remuneraciones. También se destaca la presentación de las líneas generales de los acuerdos registrados en las diferentes asambleas de accionistas.

TABLA 9. Organización y recursos humanos

\begin{tabular}{|r|l|c|c|c|}
\hline \multicolumn{2}{|c|}{ ORGANIZACIÓN Y RECURSOS HUMANOS } & SANTANDER & BBVA & CAIXABANK \\
\hline 9 & Organigrama general de la entidad & $X$ & $X$ & $X$ \\
\hline 10 & Funciones de los departamentos & & & \\
\hline 11 & Contacto de los departamentos & & & \\
\hline
\end{tabular}

Fuente: Elaboración propia.

En la tabla 9, los tres indicadores miden la transparencia respecto a los principales aspectos de la organización y recursos humanos. En este caso, las entidades analizadas muestran de manera muy clara mediante gráficos, no sólo el organigrama general de la empresa, sino que también lo desarrollan de manera más específica para que el cliente/inversor pueda ver la posición, dentro del organigrama, de los principales departamentos. Respecto a las funciones de los departamentos, no se aprecia información detallada del funcionamiento de los 


\section{Alberto Prado Román, Paola Plaza Casado, Sandra Escamilla Solano y Ana Cruz Suárez}

mismos, aunque si se hace una mención genérica de estos. Tampoco se encuentra un contacto determinado para cada departamento. Pero sí que las entidades bancarias ponen a disposición del cliente/inversor un contacto genérico, el cual está asociado al departamento de prensa correspondiente.

TABLA 10. Relaciones con los clientes y grupos de interés

\begin{tabular}{|c|c|c|c|c|}
\hline \multicolumn{2}{|r|}{$\begin{array}{c}\text { RELACIONES CON LOS CLIENTES Y GRUPOS } \\
\text { DE INTERÉS }\end{array}$} & SANTANDER & BBVA & CAIXABANK \\
\hline 12 & Horarios de atención & $X$ & $X$ & $X$ \\
\hline 13 & Webs y Portales electrónicos & $\mathrm{X}$ & $\mathrm{X}$ & $\mathrm{X}$ \\
\hline 14 & Precios e importe de los servicios y tasas & $\mathrm{X}$ & $\mathrm{X}$ & $x$ \\
\hline 15 & $\begin{array}{l}\text { Información sobre los órganos de } \\
\text { participación estructurada }\end{array}$ & $x$ & $x$ & $x$ \\
\hline 16 & $\begin{array}{llll}\text { Información } & \text { sobre } & \text { los } & \text { procesos } \\
\text { participativos } & & & \end{array}$ & $x$ & $x$ & $x$ \\
\hline 17 & $\begin{array}{l}\text { Guía de Procedimientos y Trámites y/o } \\
\text { Catálogo de Servicios }\end{array}$ & $x$ & $x$ & $x$ \\
\hline 18 & Cartas de Servicios & $x$ & $x$ & $x$ \\
\hline 19 & Quejas y sugerencias & $\mathrm{X}$ & $\mathrm{X}$ & $x$ \\
\hline
\end{tabular}

Fuente: Elaboración propia.

En esta categoría, tabla 10, se agrupan 8 indicadores sobre las relaciones de las diferentes de las entidades con sus clientes y sus respectivos grupos de interés. En este caso, las tres entidades muestran de manera muy clara toda la información necesaria para que los clientes puedan saber sus horarios de atención al público, los catálogos de los servicios que ofertan y sus respectivos precios, así como un servicio para que el público pueda presentar sus quejas y/o sugerencias a éstas.

TABLA 11. Información económico-financiera y patrimonio general

\begin{tabular}{|l|l|c|c|c|}
\hline \multicolumn{2}{|c|}{$\begin{array}{c}\text { INFORMACIÓN ECONÓMICO-FINANCIERA } \\
\text { Y PATRIMONIAL GENERAL }\end{array}$} & SANTANDER & BBVA & CAIXABANK \\
\hline 20 & Presupuestos completos & $\mathrm{X}$ & $\mathrm{X}$ & $\mathrm{X}$ \\
\hline 21 & Cuentas anuales & $\mathrm{X}$ & $\mathrm{X}$ & $\mathrm{X}$ \\
\hline 22 & Endeudamiento & $\mathrm{X}$ & $\mathrm{X}$ & $\mathrm{X}$ \\
\hline 23 & Deuda financiera & $\mathrm{X}$ & $\mathrm{X}$ & $\mathrm{X}$ \\
\hline 24 & Evolución del endeudamiento & $\mathrm{X}$ & $\mathrm{X}$ & $\mathrm{X}$ \\
\hline 25 & Ratios de endeudamiento & $\mathrm{X}$ & $\mathrm{X}$ & $\mathrm{X}$ \\
\hline 26 & Plazo de pago a proveedores & $\mathrm{X}$ & $\mathrm{X}$ & $\mathrm{X}$ \\
\hline 27 & Ratios económico-financieros & $\mathrm{X}$ & $\mathrm{X}$ & $\mathrm{X}$ \\
\hline 28 & Inventario de Bienes y Derechos & $\mathrm{X}$ & $\mathrm{X}$ & $\mathrm{X}$ \\
\hline 29 & Inmuebles & $\mathrm{X}$ & $\mathrm{X}$ & $\mathrm{X}$ \\
\hline 30 & Bienes especiales & $\mathrm{X}$ & $\mathrm{X}$ & $\mathrm{X}$ \\
\hline 31 & Informe de sostenibilidad financiera & & \\
\hline
\end{tabular}

Fuente: Elaboración propia. 


\section{Identificación de la transparencia como una herramienta de differenciación en el sector bancario español}

En la cuarta categoria, tabla 11, se hace referencia a una información fundamental para todos los clientes e inversores: la información económica-financiera y el patrimonio general de las principales entidades bancarias del mercado español. En esta categoria también se ha registrado que las entidades muestran de manera muy clara y detallada, toda la información necesaria para poder comprender la situación económica y financiera de las mismas, y asi disponer, de manera sencilla, el verdadero estado de cada una de las entidades bancarias. Además hay que resaltar, que no sólo emiten sus cuentas anuales, sino que también presentan numerosos informes detallados donde se especifica la situación de sus inversiones en inmuebles, su nivel de endeudamiento o su nivel de sostenibilidad financiera entre otros.

TABLA 12. Planificación, gestión y evaluación

\begin{tabular}{|c|l|c|c|c|}
\hline \multicolumn{2}{|c|}{ PLANIFICACIÓN, GESTIÓN Y EVALUACIÓN } & SANTANDER & BBVA & CAIXABANK \\
\hline 32 & $\begin{array}{l}\text { Datos estadísticos e indicadores } \\
\text { socioeconómicos sobre su ámbito } \\
\text { territorial o sectorial de actuación }\end{array}$ & $X$ & $X$ \\
\hline 33 & Informes de diagnóstico & $X$ & $X$ & $X$ \\
\hline 34 & Plan o Programa general de la entidad & $X$ & $X$ & $X$ \\
\hline 35 & Memoria general de la entidad & $X$ & $X$ & $X$ \\
\hline 36 & $\begin{array}{l}\text { Informe sobre el coste y rendimiento de } \\
\text { los servicios }\end{array}$ & $\begin{array}{l}\text { Informes sobre la calidad y evaluación } \\
\text { de servicios }\end{array}$ & $X$ & $X$ \\
\hline 38 & Indicadores de eficacia y eficiencia & $X$ & $X$ \\
\hline
\end{tabular}

Fuente: Elaboración propia.

Determinado el nivel de transparencia de las entidades respecto a la información económica-financiera, procedemos a determinar la transparencia dentro de la planificación, gestión y evaluación de la eficiencia de las mismas (tabla 12). Como en las demás categorias, las entidades bancarias muestran claramente sus directrices principales, sus estrategias (de manera genérica, ya que si lo hiciesen de manera detallada provocaría efectos negativos sobre las mismas), así como sus respectivos indicadores de eficacia y eficiencia. 


\section{Alberto Prado Román, Paola Plaza Casado, Sandra Escamilla Solano y Ana Cruz Suárez}

TABLA 13. Normativa y controles formales

\begin{tabular}{|c|l|c|c|c|}
\hline \multicolumn{2}{|c|}{ NORMATIVA Y CONTROLES FORMALES } & SANTANDER & BBVA & CAIXABANK \\
\hline 39 & $\begin{array}{l}\text { Normativa básica de organización y } \\
\text { funcionamiento }\end{array}$ & $\times$ & $\times$ & $\times$ \\
\hline 40 & Normativa generada por la entidad & $X$ & $X$ & $\times$ \\
\hline 41 & $\begin{array}{l}\text { Informes internos de Intervención y } \\
\text { Auditoria de Cuentas }\end{array}$ & $\times$ & $\times$ & $\times$ \\
\hline 42 & $\begin{array}{l}\text { Informes externos de auditoría y/o } \\
\text { fiscalización }\end{array}$ & $\times$ & $\times$ & $\times$ \\
\hline
\end{tabular}

Fuente: Elaboración propia.

En la penúltima categoria, tabla 13, se han fijado 4 indicadores sobre la presentación de las normativas, tanto internas como externas, a las que están sujetas las entidades bancarias, así como los controles que aplican las mismas para asegurar el funcionamiento correcto de sus servicios. En este caso, no sólo hay que señalar que las entidades no solo muestran de manera sencilla y accesible todas las normativas a las que están sujetas, sino que también presentan, de la misma manera accesible, los informes de auditoría que han sido aplicados en las mismas.

TABLA 14. Compromiso y principios generales de transparencia y buen gobierno

\begin{tabular}{|c|c|c|c|c|}
\hline \multicolumn{2}{|r|}{$\begin{array}{c}\text { COMPROMISO Y PRINCIPIOS } \\
\text { GENERALES DE TRANSPARENCIA Y } \\
\text { BUEN GOBIERNO }\end{array}$} & SANTANDER & BBVA & CAIXABANK \\
\hline 43 & $\begin{array}{l}\text { Código de Buen Gobierno o Código } \\
\text { Ético }\end{array}$ & X & $X$ & $X$ \\
\hline 44 & $\begin{array}{l}\text { Régimen sancionador en materia de } \\
\text { Buen Gobierno }\end{array}$ & $x$ & $x$ & $X$ \\
\hline 45 & $\begin{array}{lll}\text { Normativa } & \text { reguladora } & \text { de } \\
\text { transparencia } & & \end{array}$ & $x$ & $x$ & $x$ \\
\hline 46 & Web amigable y comprensible & X & $\mathrm{X}$ & $X$ \\
\hline 47 & $\begin{array}{lccc}\text { Gratuidad del acceso a la } \\
\text { información sobre transparencia }\end{array}$ & $x$ & $X$ & $x$ \\
\hline 48 & $\begin{array}{l}\text { Publicación en la web de noticias de } \\
\text { interés }\end{array}$ & $x$ & $x$ & $x$ \\
\hline 49 & Igualdad de género & * & * & * \\
\hline 50 & $\begin{array}{l}\text { Proyectos de actuación de } \\
\text { responsabilidad social }\end{array}$ & $x$ & $x$ & $x$ \\
\hline
\end{tabular}

Fuente: Elaboración propia. 


\section{Identificación de la transparencia como una herramienta de differenciación en el sector bancario español}

Finalmente en la séptima y última categoría de transparencia, tabla 14 , se ha medido el nivel de accesibilidad de los aspectos de las entidades bancarias sobre su compromiso empresarial y los principios generales respecto a la transparencia de las mismas y el buen gobierno. Es fundamental el análisis del buen gobierno de las entidades bancarias, ya que tiene una contribución muy relevante como se ha registrado en las anteriores categorias, las entidades muestran de manera clara y accesible mucha información sobre sus respectivos códigos de buen gobierno, sus respectivas normativas reguladoras de transparencia o el régimen sancionador en caso de no cumplir sus códigos de buen gobierno. También hay que destacar, los profundos y detallados informes sobre las estrategias de responsabilidad social que las entidades están aplicando, y sus objetivos futuros respecto a las mismas. Sin embargo, hay un indicador que no se cumple de manera relevante y ese es el caso del indicador 49 "Igualdad de género". En este caso, aunque todas las entidades señalan en diferentes informes que luchan contra la desigualdad de género, ninguna presenta un informe detallado sobre cuáles son las estrategias que aplicarán para combatirla. Aunque, hay que señalar que Caixabank detalla en un informe más genérico, cuáles son sus objetivos generales para luchar contra la desigualdad de género, y el Banco Santander no sólo los precisa, también de manera genérica, sino que fija el plazo en el que tiene previsto cumplirlos.

\section{DISCUSIÓN, CONCLUSIONES E IMPLICACIONES}

Como se ha demostrado, la transparencia es un aspecto caracterizado por su complejidad. Un aspecto que las empresas no solo deben ejecutar, a través de la rendición de sus cuentas, sino que debe ser considerado un aspecto inherente al desarrollo sostenible, siendo asociado a la misma identidad de las empresas. Las empresas deberán ser transparentes, motivadas por la exigencia que le mostrarán sus stakeholders para poder acceder a la información de las mismas (Rivero, 2005). Además, las empresas deberán entender que el nivel de transparencia que muestran se considera un elemento fundamental en la generación de confianza (Arredondo et al., 2014). Sin embargo, no por ser más transparentes la confianza de sus stakeholders aumentará. Por ello, las empresas no sólo tendrán que ser transparentes sino que tendrán que convencer a la sociedad, y al resto de sus stakeholders, que lo son porque eso se reflejará en confianza por parte de éstos.

Uno de los sectores más criticados por su falta de transparencia en España es el sector bancario. La sociedad, como integrante de sus stakeholders, cree que las entidades bancarias son muy pocos transparentes. Que las mismas, lejos de 


\section{Alberto Prado Román, Paola Plaza Casado, Sandra Escamilla Solano y Ana Cruz Suárez}

facilitar información sobre los diferentes aspectos empresariales de éstas no los presentan, o si lo hacen, son muy dificiles de localizar. ${ }^{11}$

Por ello, nuestra investigación se centró en determinar, si esa desconfianza por parte de la sociedad es legítima o simplemente es producto de los escándalos financieros pasados, demostrando que los casos negativos extraordinarios ocurridos durante los últimos años han afectado enormemente a la imagen de las entidades bancarias analizadas. Los resultados demostraron que no sólo las principales entidades bancarias españolas son muy transparentes, sino que todas ellas llegan a cumplir con 47 de los 50 indicadores analizados. Es decir, que estas entidades cumplen el $94 \%$ de los indicadores de transparencia propuestos por esta investigación.

Dentro de los indicadores que cumplen, destaca el cumplimiento de todos los indicadores referentes a la información económica y financiera de las mismas. Estos indicadores representan el $24 \%$ del total. Además, los informes presentados por las principales entidades bancarias españolas muestran informaciones muy detalladas y especificas sobre los principales aspectos que afectan a sus respectivas situaciones económicas y financieras, así como los mismos informes pero de años anteriores para que los clientes/inversores puedan determinar las respectivas evoluciones. Los únicos indicadores que no se cumplen están englobados dentro de las categorias de "Organización y Recursos Humanos" y de "Compromisos y Principios Generales de Transparencia y Buen Gobierno". El primero está formado por 3 indicadores, y aunque las tres entidades cumplen el indicador de accesibilidad al organigrama general de la entidad, no cumplen el de accesibilidad a las funciones de los departamentos y al contacto de los mismos. Sin embargo, consideramos que la relevancia de éstos en el nivel de transparencia de las entidades bancarias, es relativa. Dicha razón reside en que aunque éstas no detallan de manera especifica todas las funciones de cada departamento, sí que las precisan de manera general. Además, sí que presentan un contacto general para poder contactar con las empresas.

Respecto a la segunda categoría, ésta está formada por 8 indicadores, de los cuáles las tres entidades cumplen 7 , es decir el $87.5 \%$ de dicha categoría. El indicador que no cumplen es el que hace referencia a la publicación de informes sobre estrategias para alcanzar la igualdad de género. Si bien es cierto que las tres entidades señalan, en numerosos informes, que no están de acuerdo con la desigualdad de género y que están trabajando para eliminarla. Además, Caixabank detalla de manera más específica sus objetivos principales en la estrategia de la igualdad de género, y el Banco Santander incluso fija la fecha en la que pretende que esos objetivos se cumplan.

\footnotetext{
${ }^{11}$ Principales resultados recogidos en el Segundo Barómetro de la Transparencia de España 2016 desarrollado por Acreditra.
} 


\section{Identificación de la transparencia como una herramienta de differenciación en el sector bancario español}

Por todo ello, podemos determinar que las principales entidades bancarias de España son muy transparentes. Ello nos indica que el gran problema del sistema bancario español no es su falta de transparencia, sino su incapacidad para proyectar esa imagen al mercado. Esta incapacidad está fuertemente condicionada por los numerosos escándalos financieros pasados que han provocado que la sociedad recele de todas las entidades financieras, incluidas las bancarias. Por ello, no puede ser suficiente que dichas entidades se limiten a presentar sus respectivos informes sobre todos los aspectos relevantes de las mismas, sino que tienen que realizar campañas de marketing sólidas para convencer a los usuarios de lo transparentes que son. No es tan importante ser transparentes, sino que la gente sepa que la empresa es transparente. Ya que en ese caso, la sociedad procederá a volver a confiar en dichas entidades y por ello a legitimarlas, lo que repercutiría positivamente en sus futuras estrategias empresariales.

Durante la realización de este trabajo se ha presentado como limitaciones, en primer lugar, que esta investigación realiza una función analítica y de diagnóstico, frente a otras funciones desarrolladas por Acreditra, como son la adecuación y solicitud de acreditación. En segundo lugar, únicamente se ha analizado el nivel de transparencia de las principales entidades bancarias que cotizan en el IBEX - 35 del sector bancario español, sin tener en cuenta el resto de las entidades bancarias que operan en España. Por ello, en las futuras líneas de investigación se procederá a determinar el nivel de transparencia de todas entidades españolas bancarias que coticen en el IBEX - 35, extendiendo posteriormente dicho análisis a todas las entidades que operan en sector bancario español independientemente de su nacionalidad. 


\section{Alberto Prado Román, Paola Plaza Casado, Sandra Escamilla Solano y Ana Cruz Suárez}

\section{BIBLIOGRAFÍA}

Acreditra (2019). "Principales resultados recogidos en el Segundo Barómetro de la Transparencia de España 2016 desarrollado por Acreditra". Disponible en: http://acreditra.com/barometro-de-la-transparencia-de-espana-2016/

Aguilera, R. F. y Cuervo-Cazurra, A. (2004). "Codes of good governance worldwide: what is the trigger?", Organizations Studies, 25 (3): 417-446.

Alejos, C. L. (2015). "Transparencia: fuente de confianza en la empresa", Cuadernos de la Cátedra "la Caixa" de Responsabilidad Social de la Empresa y Gobierno Corporativo, 27: 1-32.

Alvarado, A.; Bigné, E. y Currás, P. (2011). "Perspectivas teóricas usadas para el estudio de la Responsabilidad Social Empresarial: Una clasificación con base en su racionalidad", Estudios Gerenciales, 27 (118): 115-137.

Arredondo, T.F.G.; De la Garza, G.J. y Vázquez, P.J.C. (2014). "Transparencia en las organizaciones, una aproximación desde la perspectiva de los colaboradores", Estudios Gerenciales, 30: 408-418.

Banco Santander (2018). "Memoria corporativa". Disponible en: https://wwww.santander.com/csgs/Satellite/CFWCSancomQP01/es_ES/Co rporativo/Accionistas-e-Inversores.html)

Bañon, A.; Guillen, M. y Ramon, N. (2011). "La empresa ética y responsable", Universia Business Review, 30: 32-43.

Baraibar Diez, E. y Luna Sotorrio, L. (2018). "The mediating effect of transparency in the relationship between corporate social responsibility and corporate reputation", RBGN-Revista Brasileira de Gestao de Negocios, 20 (1): 5-21.

Baraibar Diez, E. y Luna Sotorrío, L. (2012). "The influence of transparency of university social responsibility in the creation of reputation", Regional and Sectoral Economics Studies, 12 (3): 21-31.

Barea, J. (2004). "La necesidad de la transparencia en la gestión pública", Auditoria Pública, 33: 13-24.

Baumann, D. (2016). "Corporate social responsibility in a globalizing world", Business Ethics Quarterly, 26 (1): 137-141.

BBVA (2018). "Memoria corporativa". Disponible en: https://accionistaseinversores.bbva.com/informacion-financiera/informesfinancieros/\#2018 


\section{Identificación de la transparencia como una herramienta de differenciación en el sector bancario español}

Caixabank (2018). "Memoria corporativa". Disponible en: https://www.caixabank.com/informacionparaaccionistaseinversores/gobie rnocorporativo/juntageneralaccionistas/2013_es.html

Canales Aliende, J.M. y Romero Tarín, A. (2017). "Algunas consideraciones sobre la transparencia pública y el buen gobierno", Revista Española de Transparencia, 5: $115-127$.

Cortés, F (2008). "Ética empresarial y responsabilidad social empresaria", Boletín Económico de ICE, 2936.

Deegan, C. y Rankin, M. (1996). "Do Australian Companies Report Environmental News Objectively? An Analysis of Environmental Disclosures by Firms Prosecuted Successfully by the Environmental Protection Authority", Accounting, Auditing \& Accountability Journal, 9 (2): 50-67.

Diez, F.; Blanco, A.; Cruz, A. I. y Prado, C. (2014). "Efecto de la Responsabilidad Social Empresarial sobre la Legitimidad de las Empresas", Anuario Jurídico y Económico Escurialense, 47.

Escamilla, S.; Jiménez, I. y Prado, C. (2013). "La responsabilidad social empresarial, una forma de crear valor", Editorial Académica Española.

Escamilla, S.; Plaza, P. y Flores, S. (2016). "Análisis de la divulgación de la información sobre la responsabilidad social corporativa en las empresas de transporte público urbano en España", Revista de Contabilidad - Spanish Accounting Review, 19 (2): 195-203.

Freeman, E. (1984). Strategic management. A stakeholder approach. Mershfield: Publishing inc.

Gálvez, R. M. M.; Caba, P. M. C. y López, G. M. (2009). "La Transparencia On-line de las ONG Españolas", Revista Española del Tercer Sector, 13 (SeptiembreDiciembre): 63-86.

Garcia, L. M. J.; Amat, O. y Rocafort, A. (2016). "Self-perception of ethical behaviour: The case of listed Spanish companies", Intangible Capital, 12 (1): 319-335.

Garriga, E. y Melé D. (2004). "Corporate social responsibility theories: mapping the territory", Journal of Business Ethics, 53: 51-71.

GESEM Asesoramiento Financiero EAFI, S.L. (2012). "Rescate para el Sistema
Financiero
Español".
Disponible
en:

http://www.gesemav.com/informes/historico/5-

Rescate\%20para\%20el\%20sistema\%20financiero\%20espa\%C3\%B1ol.pdf 


\section{Alberto Prado Román, Paola Plaza Casado, Sandra Escamilla Solano y Ana Cruz Suárez}

Gibbins, M.; Richardson, A. y Waterhouse, J. (1990). "The management of corporate financial disclosures: Opportunism, ritualism, policies and processes", Journal of Accounting Research, 28 (1): 121-143.

Gray, R.; Kouhy, R. y Lavers, S. (1995). "Corporate Social and Environmental Reporting: A Review of the Literature and a Longitudinal Study of UK Disclosure", Accounting, Auditing \& Accountability Journal, 8 (2): 47-77.

Hooghiemstra, R. (2000). "Corporate Communication and Impression Management: New Perspectives Why Companies Engage in Corporate Social Reporting", Journal of Business Ethics, 27 (1/2): 55-68.

Kliksberg, B. (2003). "La ética importa", Revista Venezolana de Gerencia, 8 (24): 661-665.

Kliksberg, B (2006). "Ética empresarial: ¿Moda o demanda imparable?", Fundación Carolina, Working Papers.

Kolk, A. (2003). "Trends in Sustainability Reporting by the Fortune Global 250", Business Strategy and the Environment, 12 (5): 279-291.

Lev, B. (1992). "Information disclosure strategy", California Management Review, 34 (Summer): 9-32.

Lipovetsky, G. (2006). Metamorfosis de la cultura liberal. Ética, medios de comunicación, empresa. Barcelona: Anagrama.

Marcuello, C.; Bellostas, A.; Marcuello, C. y Moneva, J. M. (2007). "Transparencia y rendición de cuentas de cuentas en las empresas de inserción", Revista de Economía Pública, Social y Cooperativa, 59 (91-122).

Maudos, J. (2012). "El impacto de la crisis en el sector bancario español", Cuadernos de Información Económica, 226 (Enero/Febrero): 155-163.

Méndez, M. (2005). "Ética y responsabilidad social corporativa. Ética y Economía", ICE, 823: 141-155.

Navarro García, F. (2008). Responsabilidad Social Corporativa: Teoría y Práctica. ESIC EDITORIAL

Observatorio de Responsabilidad Social Corporativa (2019). "La gestión de los riesgos de corrupción en las empresas del IBEX 35". Disponible en: https://observatoriorsc.org/wp-content/uploads/2018/06/Informeejecutivo-corrupcion-lbex-35.pdf 


\section{Identificación de la transparencia como una herramienta de differenciación en el sector bancario español}

Ochsenius Robinson, I. (2017). "La transparencia como control y beneficio de una contratación pública presente", Revista Española de Transparencia, 4: 110-124.

Olmedo, I. y Martínez, I. M. (2011). "Medida de la reputación empresarial en PYMES de servicios", Revista Europea de Dirección y Economía de la Empresa, 20 (3): 77-102.

Piechocki, R. (2004). "Transparency of annual sustainability reports", Corporate Reputation Review, 7 (2): 107-123.

Porter, M. E. y Kramer, M. R. (2003). "La filantropía empresarial como ventaja competitiva", Harvard Deusto Business Review, 112: 7-20.

Prado, C. (1995). "Globalización de la empresa e integración de los enfoques no organizativos en la dirección", RACEF: 8.

Rivero, T.P. (2005). "Responsabilidad Social y Gobierno Corporativo: Información y Transparencia", Revista Asturiana de Economía, 34: 9-29.

Ruiz, M.; Tirado, P. y Morales, A. (2008). "Transparencia y calidad de la información económico-financiera en las entidades no lucrativas. Un estudio empírico a nivel andaluz", Revista de Economía Pública, Social y Cooperativa, 63: 253-274.

Skinner, D. (1994). "Why firms voluntarily disclose bad news", Journal of Accounting Research, 32 (Spring): 38-60.

Toms, J. S. (2002). "Firms resources, quality signals and the determinants of corporate environmental reputation: Some UK Evidence", The British Accounting Review, 34 (3): 257-282.

Transparencia Internacional España (2019). "Índices Transparencia España". Disponible en: https://transparencia.org.es/indices-transparencia-espana/

Valderrama, J. A. (2007). "Reflexiones en torno a la RS de las empresas", Teoria y Praxis, 3: 125-134.

Villanueva, E. (2011). "Aproximaciones conceptuales a la idea de transparencia", en Instituto Federal de Acceso a la Información Pública (IFAI), Transparencia: libros, autores e ideas. Coyoacán: IFAI, 57-65

Watson, A.; Shrives, P. y Marston, C. (2002). "Voluntary disclosure of accounting ratios in the UK", British Accounting Review, 34. 289-313. 